\title{
AVALIAÇ̃̃O DA MICOFLORA E OCORRÊNCIA DE MICOTOXINAS EM CASCAS DE AMENDOIM EM DIFERENTES ESTÁGIOS DE MATURAÇÃO DA VAGEM
}

\author{
Evaluation of mycoflora and occurrence of mycotoxins in peanut hulls at \\ different pod maturation stages
}

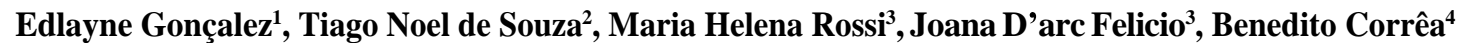

\begin{abstract}
RESUMO
As cascas de amendoim (Arachis hypogaea L.) são de grande importância para confecção de cama de frangos, de gado de leite e como fonte de fibras para ruminantes, portanto a elucidação dos mecanismos de contaminação por fungos toxigênicos e por micotoxinas em amendoim é imprescindível, especialmente para que medidas preventivas possam ser tomadas. Realizou-se, este trabalho, em Junqueirópolis, Estado de São Paulo, Brasil. Os principais fungos isolados nas cascas de amendoim foram Fusarium ssp. (78,75 \%), Rhizopus ssp. (14,1\%) e A. flavus (11,75\%). No solo foram isolados Penicillium spp., Fusarium spp. e Aspergillus flavus, entre outros. Aflatoxinas foram detectadas em amostras de cascas de amendoim a partir do estágio de granação em concentrações que variaram de $5,42 \mu \mathrm{g} / \mathrm{kg}$ a 218,52 $\mu \mathrm{g} / \mathrm{kg}$. Ácido ciclopiazônico e fumonisinas $\mathrm{B}_{1}$ e $\mathrm{B}_{2}$ não foram detectadas. A presença de $A$. flavus e aflatoxinas nas amostras, revela a importância de um controle das cascas de amendoim antes de sua utilização. Boas práticas agrícolas são indicadas para região, uma vez que a contaminação das vagens ocorreu antes da colheita.
\end{abstract}

Termos para indexação: Aflatoxinas, ácido ciclopiazônico, fumonisinas, Aspergillus flavus, amendoim, Arachis hypogaea L.

\begin{abstract}
Peanut (Arachis hypogaea L.) hulls are very important because they are used as litter to poultry and dairy cattle and as fiber source to cattle. The elucidation of the peanuts contamination mechanisms by toxigenic fungi and their mycotoxins is vital, specially for prevention measurements. The peanuts total mycoflora and mycotoxin contamination were analyzed in plants sampled in Junqueirópolis, in São Paulo State (Brazil) at different stages of the pod maturity. The prevalent mycoflora in peanut hulls were Fusarium spp., Rhizopus spp. and Aspergillus flavus. In soil under the peanut crop, the genus Penicillium spp., Fusarium spp. and A. flavus were detected. Aflatoxins were detected in peanut hull samples since filling pod stage in concentrations from $5.42 \mu \mathrm{g} / \mathrm{kg}$ to $218.52 \mu \mathrm{g} / \mathrm{kg}$. Cyclopiazonic acid and fumonisins were not detected. The A. flavus presence and the detection of aflatoxins indicate the importance of quality control of peanut hulls before their utilization, and the adoption of agricultural practices showed to avoid the contamination since the peanut pods contamination happened before the harvest.
\end{abstract}

Index terms: Aflatoxin, Aspergillus flavus, cyclopiazonic acid, fumonisin, peanut, Arachis hypogaea L.

(Recebido em 12 de setembro de 2007 e aprovado em 7 de maio de 2008)

\section{INTRODUÇÃOO}

O amendoim (Arachis hypogaea L.) é um importante produto agrícola que se adapta a uma larga faixa climática dentro de regiões tropicais e subtropicais (FAGUNDES, 2002). No Brasil, $83,2 \%$ da produção nacional realiza-se no Estado de São Paulo (FAGUNDES, 2002), sendo que a safra 2005/06 ultrapassou 400 mil toneladas (MARTINS, 2006a). O saldo da balança comercial paulista para grãos e produtos de amendoim foi de US\$ 54,4 milhões em 2005 (MARTINS, 2006b). Já a safra 2006/2007, quando comparada a de 2005/06, houve uma redução de $23 \%$ na produção e de $12 \%$ na área plantada (MARTINS, 2007).
$\mathrm{O}$ amendoim é um produto muito susceptível à infestação por fungos e contaminação por micotoxinas. Sua contaminação pelos Aspergillus flavus e $A$. parasiticus, fungos produtores de aflatoxinas, pode ocorrer antes ou após a colheita (MPHANE et al., 2004). A espécie Aspergillus flavus pode, também, produzir o ácido ciclopiazônico que é um ácido indólico que ocorre naturalmente em produtos agrícolas, tais como, amendoim e milho e, muitas vezes, como um co-contaminante com as aflatoxinas (LISKER et al., 1993). Outras micotoxinas foram detectadas em amendoim, tais como ocratoxina A, zearalenona e fumonisina $\mathrm{B}_{1}$ (SANGARE-TIGORE et al., 2006).

\footnotetext{
'Biológa, Doutora - Centro de Pesquisa e Desenvolvimento de Sanidade Animal/CPDSA - Instituto Biológico/IB - Avenida Conselheiro Rodrigues Alves, 1252 -04014-002 - São Paulo, SP - goncalez@biologico.sp.gov.br

${ }^{2}$ Graduando em Ciências Farmacêuticas - Centro de Pesquisa e Desenvolvimento de Sanidade Animal/CPDSA - Instituto Biológico/IB - Avenida Conselheiro Rodrigues Alves, 1252 - 04014-002 - São Paulo, SP - jholynt@yahoo.com.br

${ }^{3}$ Químicas, Doutoras - Centro de Pesquisa e Desenvolvimento de Sanidade Animal/CPDSA - Instituto Biológico/IB - Avenida Conselheiro Rodrigues Alves, 1252 - 04014-002 - São Paulo, SP - rossi@biologico.sp.gov.br; felicio@biologico.sp.gov.br

${ }^{4}$ Biomédico, Doutor - Instituto de Ciências Biomédicas/ICB - Universidade de São Paulo/USP - Avenida Professoror Lineu Prestes, s/n - 05508-900 São Paulo, SP - correabe@usp.br
} 
As cascas de amendoim são utilizadas, tanto no Brasil como em outros países como cama de frango, cama para gado leiteiro confinado, como fonte de fibras para ruminantes e sua farinha é utilizada como fonte de fibras de baixa caloria em vários produtos alimentícios para o homem, desde que a presença de aflatoxinas seja controlada (COLLINS \& POST, 1981; LIEN et al., 1998).

Neste trabalho foi verificada a presença de micotoxinas (aflatoxinas, ácido ciclopiazônico e fumonisinas) e identificada a microbiota fúngica em amostras de cascas de amendoim, em diferentes estágios de maturação e solo. Essas cascas são provenientes de grãos de amendoim contaminados com aflatoxinas e foram utilizadas como cama de frango, em granjas situadas no Estado de São Paulo, Brasil.

\section{MATERIALE MÉTODOS}

\section{Local de Coleta}

Realizou-se, o estudo, em Junqueirópolis, Estado de São Paulo, Brasil. A semente de amendoim utilizada no plantio foi da variedade Runner IAC 886, plantada no mês de dezembro de 2004 e o amendoim foi colhido em março de 2005. Foram realizadas 05 coletas nos seguintes estágios de maturação dos frutos do amendoim: $1^{\mathrm{a}}$ coleta: frutos recém-formados; $2^{a}$ coleta: frutos em início de granação; 3 Coleta: frutos em granação; $4^{a}$ Coleta: frutos maduros; 5 Coleta: frutos após a secagem. Amostras de solo foram coletadas em cada estágio de maturação do amendoim (coletas de 1 a 4 ).

\section{Amostragem do amendoim e do solo}

A região do plantio foi dividida em 10 parcelas uniformes de $80 \mathrm{~m}^{2}$, sendo que para cada coleta foram escolhidas, ao acaso, 5 parcelas. Para cada um dos períodos de coleta foram colhidas 5 amostras, contendo em torno de $1,0 \mathrm{~kg}$ de amendoim e 05 amostras de solo de aproximadamente 1,0 kg (DELP et al., 1986).

\section{Isolamento e identificação dos fungos das casca}

De cada uma das 25 amostras dos frutos de amendoim foram retirados, aproximadamente, $30 \mathrm{~g}$ para desinfecção em solução de hipoclorito de sódio 2,0 \%, por 3 minutos. Após a desinfecção, foi separada a casca dos grãos do amendoim e lavados com água estéril. Foram utilizadas, para cada amostra, 3 placas de Petri contendo cascas de amendoim, semeados diretamente em meio ágar DRBC e AFPA (PITT et al., 1979). Todas as placas foram incubadas a $25^{\circ} \mathrm{C}$ por 5 dias e os resultados expressos em porcentagem do total de cascas inoculadas contaminadas por fungos.
Em seguida, colônias de diferentes tipos morfológicos foram isoladas em ágar Sabouraud dextrose e submetidas à identificação, de acordo com os compêndios recomendados para cada gênero (BARNETT \& HUNTER, 1972; NELSON et al., 1983; RAPER \& FERNEL, 1965).

\section{Isolamento e identificação dos fungos do solo}

Foram coletadas um total 20 amostras de solo obtidas na parte superficial ( $0-5 \mathrm{~cm}$ de profundidade), contendo aproximadamente $1,0 \mathrm{~kg}$ cada. Cada uma das amostras foi homogeneizada e analisada de acordo com o método descrito por Swanson et al. (1992), utilizando o meio Martin para o isolamento da microbiota. As placas foram incubadas a $25^{\circ} \mathrm{C}$ por dez dias. As colônias fúngicas foram identificadas de acordo com os compêndios recomendados para cada gênero (BARNETT \& HUNTER, 1972; NELSON et al., 1983; RAPER \& FERNEL, 1965).

\section{Determinação da atividade de água}

As atividades de água das amostras de cascas de amendoim e do solo foram determinadas por meio do aparelho AQUALAB CX-2 (Decagon Devices Inc.).

\section{Determinação de Micotoxinas}

\section{Aflatoxinas}

Para extração e purificação das aflatoxinas presentes nas cascas de amendoim em diferentes estágios de maturação, foi utilizado o método AOAC Official Mehod 991.31 (AOAC, 2000), modificado pela empresa Vicam. Foram utilizadas $25 \mathrm{~g}$ de cascas trituradas com $10 \mathrm{~g}$ de $\mathrm{NaCl}$ e adicionados $250 \mathrm{~mL}$ de metanol: água (60:40 v/v). Após agitação por 30 minutos em agitador mecânico horizontal, o conteúdo foi filtrado em papel de filtro e 20 $\mathrm{mL}$ do extrato foi diluído com $20 \mathrm{~mL}$ de água destilada. Após nova filtração, $4,0 \mathrm{~mL}$ do extrato $(4,0 \mathrm{~mL}=0,2 \mathrm{~g}$ da amostra) foram transferidos para uma coluna de imunoafinidade (Aflatest, Vicam Somerville, MA, USA) a um fluxo de cerca de 1-2 gotas/segundo até que o ar passasse pela coluna. Em seguida, a coluna foi lavada com 5,0 mL de água destilada a um fluxo de cerca de 2 gotas/ segundo e eluída com 1,0 mL de metanol grau CLAE a um fluxo de cerca de 1-2 gotas/segundo. As aflatoxinas $\mathrm{B}_{1}, \mathrm{~B}_{2}$, $\mathrm{G}_{1}$ e $\mathrm{G}_{2}$ presentes no extrato metanólico foram quantificadas por CLAE.

Condições Cromatográficas: $\mathrm{O}$ equipamento utilizado foi da marca Shimadzu, modelo LC6AD (Kyoto, Japan) com detector de UV, modelo SPD 6AV e detector espectrofluométrico RF 551 (Shimadzu, Kyoto, Japan) e monitorados pelo programa Shimadzu class VP 5.02. A fase 
móvel utilizada foi metanol:acetonitrila:ácido fosfórico 0,1 $\%(24: 24: 52 \mathrm{v} / \mathrm{v} / \mathrm{v})$, fluxo de $1,0 \mathrm{~mL} / \mathrm{min}$, detector de fluorescência (364 nm de excitação e $440 \mathrm{~nm}$ de emissão), coluna ODS C18 4,6×250 mm de 5,0 micron (Shimadzu) e temperatura do forno de $30^{\circ} \mathrm{C}$ (CHAN et al., 2004). O método de quantificação das amostras foi padrão externo, utilizando-se curva de calibração com 5 pontos para cada aflatoxina. Os pontos utilizados foram: 8,$09 ; 11,32 ; 16,18$; 22,65 e $32,36 \mathrm{ng} / \mathrm{mL}$ para $\mathrm{AFB}_{1} ; 1,00 ; 1,40 ; 2,00 ; 2,80$ e 4,00 $\mathrm{ng} / \mathrm{mL}$ para $A F B ; 2,54 ; 3,55 ; 5,08 ; 7,11$ e $10,16 \mathrm{ng} / \mathrm{mL}$ para $\mathrm{AFG}_{1}$ e 1,$92 ; 2,67 ; 3,84 ; 7,11 ; 7,68 \mathrm{ng} / \mathrm{mL}$ para $\mathrm{AFG}_{2}$. O limite de quantificação foi determinado como sendo o mínimo de toxina possível de ser quantificada no comprimento de onda de $364 \mathrm{~nm}$ de excitação e $440 \mathrm{~nm}$ de emissão. O limite de detecção foi estabelecido como sendo o pico da concentração da toxina 3 vezes superior à linha de base do cromatograma. Os limites de quantificação e detecção foram determinados : 3,50 e 2,00 ng/mL para $\mathrm{AFB}_{1}$; 0,10 e $0,05 \mathrm{ng} / \mathrm{mL}$ para $\mathrm{AFB}_{2} ; 2,00$ e $1,00 \mathrm{ng} / \mathrm{mL}$ para $\mathrm{AFG}_{1}$ e 0,10 e $0,05 \mathrm{ng} / \mathrm{mL}$ para $\mathrm{AFG}_{2}$, respectivamente.

\section{Confirmação da presença de aflatoxinas}

As amostras que apresentaram aflatoxinas detectáveis foram confirmadas segundo Tarin et al. (2004), em que $500 \mu \mathrm{L}$ da amostra foi seca sob nitrogênio e diluída em $50 \mu \mathrm{L}$ de ácido trifluoroacético (TFA) e $200 \mu \mathrm{L}$ de hexano a $40{ }^{\circ} \mathrm{C}$. A solução foi agitada por 15 minutos, seca sob nitrogênio e redissolvida com $200 \mu \mathrm{L}$ de acetonitrila:água $(3: 7 \mathrm{v} / \mathrm{v})$ e analisada por CLAE.

\section{Ácido ciclopiazônico}

Para extração e purificação do ácido ciclopiazônico (ACP), foi utilizado o método de Urano et al. (1992) com algumas modificações. $25 \mathrm{~g}$ de cada amostra, foram trituradas e acrescidos de $200 \mathrm{~mL}$ de metanol:bicarbonato de sódio $2 \%$ em água (7:3). Após agitação por 30 minutos em agitador mecânico horizontal, o conteúdo do frasco foi filtrado em papel de filtro. Uma alíquota de $100 \mathrm{~mL}$ do extrato foi transferido para um funil de separação e adicionados $100 \mathrm{~mL}$ de hexano. A solução foi agitada e o hexano descartado e em seguida, foram adicionados $50 \mathrm{~mL}$ de uma solução aquosa de $\mathrm{KCl} 10 \%$. Essa solução foi acidificada com 2,0 mL de $\mathrm{HCl} 6 \mathrm{~N}$. O ACP foi extraído da solução com $50 \mathrm{~mL}$ de clorofórmio por duas vezes. Após adicionar sulfato de sódio anidro, a fração clorofórmica foi filtrada e evaporada até a secura em evaporador rotativo. O resíduo foi ressuspendido em $10 \mathrm{~mL}$ de clorofórmio e transferidos para uma coluna de extração de fase sólida Micotox ${ }^{\circledR}$ MS2300 (Micotox Ltda, Bogotá D.C., Colômbia) a um fluxo de 2,0 $\mathrm{mL} / \mathrm{min}$, até que o ar passasse pela coluna.
Completada a operação, a coluna foi lavada com $10 \mathrm{~mL}$ de éter etílico, $10 \mathrm{~mL}$ de clorofórmio/acetona $(1: 1 \mathrm{v} / \mathrm{v})$ seguido de $10 \mathrm{~mL}$ de clorofórmio/metanol $(95: 5 \mathrm{v} / \mathrm{v})$. O ACP foi eluído com $10 \mathrm{~mL}$ de clorofórmio/metanol (75:25 v/v) a um fluxo de $2 \mathrm{~mL} / \mathrm{min}$. $\mathrm{O}$ extrato foi evaporado sob nitrogênio até a secura e diluído em 1,0 mL de metanol grau CLAE. O extrato foi armazenado em temperatura de congelamento até o momento da quantificação.

Quantificação de ácido ciclopiazônico por CLAE: O ácido ciclopiazônico foi quantificado por CLAE, tendo como fase móvel acetonitrila:tampão acetato de amônio $(0,05 \mathrm{M}, \mathrm{pH}$ $5,0)(8: 2 \mathrm{v} / \mathrm{v})$, fluxo de $0,6 \mathrm{~mL} / \mathrm{min}$, detector de UV em 284 nm (LOSITO et al., 2002), coluna ODS C8 4,6 x 250 mm de 5,0 micron (Shimadzu). O método de quantificação das amostras foi padrão externo, utilizando-se curva de calibração com 5 pontos $(0,611 ; 1,223 ; 2,446 ; 4,892$ e 9,798 $\mu \mathrm{g} / \mathrm{mL})$. O limite de quantificação foi determinado como sendo o mínimo de toxina possível de ser quantificada, no comprimento de onda de $284 \mathrm{~nm}$. O limite de detecção foi estabelecido como sendo o pico da concentração da toxina 3 vezes superior à linha de base do cromatograma. Os limites de quantificação e detecção foram: $0,03 \mu \mathrm{g} / \mathrm{mL}$ e $0,005 \mu \mathrm{g} / \mathrm{mL}$, respectivamente.

\section{Fumonisinas}

Para extração e purificação das fumonisinas $\mathrm{B}_{1} \mathrm{e}$ $\mathrm{B}_{2}$, foi utilizado o método descrito por Visconti et al. (2001) com algumas modificações. $10 \mathrm{~g}$ de cada amostra, foram trituradas e acrescidos $100 \mathrm{~mL}$ de acetonitrila:metanol:água (25:50:50 v/v/v). Após agitação por 20 minutos, em agitador mecânico horizontal, as amostras foram centrifugadas a $2500 \mathrm{rpm}$ por 10 minutos, e o sobrenadante filtrado em papel de filtro. As operações foram repetidas novamente para o precipitado, e os filtrados recolhidos. $20 \mathrm{~mL}$ do filtrado foram diluídos com $20 \mathrm{~mL}$ de tampão fosfato (PBS) $\mathrm{pH} 7,0$, agitado e filtrado em papel de filtro de microfibra e, em seguida, coletados $10 \mathrm{~mL}$ para purificação em coluna de imunoafinidade (Fumonitest, Vicam, Somerville, MA, USA) com fluxo de 2-3 mL por minuto. As fumonisinas foram eluídas com 1,5 mL de metanol grau CLAE e evaporado até a secura e mantido em congelador até o momento da quantificação.

Quantificação de fumonisinas por CLAE: $200 \mu \mathrm{L}$ da amostra foram diluídos em $50 \mu \mathrm{L}$ de reagente OPA (40 mg de ortoftaldeído em 1,0 mL de metanol, diluídos em 5,0 mL de solução $0,1 \mathrm{M}$ de teraborato de sódio, acrescidos de 50 $\mu \mathrm{L}$ de mercaptoetanol) em temperatura ambiente por 2 minutos. O produto dessa reação foi analisado por CLAE em sistema isocrático de fase reserva usando uma coluna C18 (4,6 x 150 mm), 5,0 micron (J.T. Baker) e comprimento 
de onda $440 \mathrm{~nm}$ de emissão e $335 \mathrm{~nm}$ de excitação. A fase móvel utilizada foi acetonitrila:água:ácido acético (520:480:5), com fluxo de 1,0 $\mathrm{mL} / \mathrm{min}$ e temperatura do forno de $30{ }^{\circ} \mathrm{C}$. O método de quantificação das amostras foi padrão externo, utilizando curva de calibração com 4 pontos para cada fumonisina $\left(2,0 ; 1,0 ; 0,5 ; \mathrm{e} 0,25 \mu \mathrm{g} / \mathrm{mL}\right.$ para $\mathrm{FB}_{1} \mathrm{e}$ 1,$0 ; 0,5 ; 0,25$ e $0,125 \mu \mathrm{g} / \mathrm{mL}$ para $\mathrm{FB}_{2}$ ). O limite de quantificação foi determinado como sendo o mínimo de toxina possível de ser quantificada no comprimento de onda de $440 \mathrm{~nm}$ de emissão e $335 \mathrm{~nm}$ de excitação. O limite de detecção foi estabelecido como sendo o pico da concentração da toxina 3 vezes superior à linha de base do cromatograma. Os limites de quantificação e detecção foram: 0,25 e $0,025 \mu \mathrm{g} / \mathrm{mL}$ para $F_{1}$ e 0,125 e $0,0125 \mu \mathrm{g} / \mathrm{mL}$ para $\mathrm{FB}_{2}$, respectivamente.

\section{Análise estatística}

Estimaram-se modelos de regressão linear, a fim de permitir que diversas variáveis explicativas respondessem pelas variáveis independentes (A. flavus nas cascas e no solo e atividade de água das cascas e do solo). As variáveis dependentes foram as micotoxinas presentes nas cascas do amendoim nos diferentes estágios de maturação. Para estimar os modelos, usou-se o procedimento REG do SAS ${ }^{\circledR}$ (SAS INSTITUTE, 2008).

\section{RESULTADOS E DISCUSSÃO}

A partir das análises microbiológicas das 25 amostras de cascas de amendoim, a população fúngica foi constituída pelos seguintes fungos: Fusarium ssp. (78,75 $\%)$, Rhizopus ssp. (14,1\%), A. flavus (11,75\%), Absidia ssp. (8\%), A. niger (2,5\%), Trichoderma ssp. (0,8\%), Penicillium ssp. (0,7 \%), Scopolariopsis ssp. $(0,15 \%)$ e A. candidus $(0,13 \%)$. Outros autores, estudando a microbiota fúngica do amendoim, relataram os gêneros Aspergillus spp., Fusarium spp. e Penicillium spp., como os principais contaminantes (MPHANE et al., 2004). A maior incidência da espécie A. flavus (49\%) foi constatada no estágio de granação dos frutos, seguido pelo fruto já maduro $(8,8 \%)$. A citada espécie também foi isolada em 1,2\% das cascas de frutos recém-formados (Tabela 1 e Figura 1).

Os valores médios de atividade de água variaram de 0,99 a 0,61 , sendo que no estágio de granação $\left(A_{a}=0,96\right)$ e no fruto maduro $\left(A_{a}=0,90\right)$ esse fator foi importante para contaminação por A. flavus ( $\mathrm{p}<0,05$ e $\mathrm{r}^{2}=0,62$ ). Nas cascas de frutos após a secagem, A. flavus não foi isolado, sendo que nessas amostras a média de atividade de água foi de 0,61 , valor abaixo do considerado ideal para do crescimento do fungo, que é de 0,8 (SMITH \& MOSS, 1985). Durante a formação da vagem do amendoim e início da granação, os frutos são constituídos por uma grande quantidade de água (tabela 1). Nesses estágios, foi encontrada uma alta incidência de Fusarium spp., fungo melhor adaptado a substratos com maior disponibilidade de água (HORN, 2005).

O estudo das 20 amostras de solo resultou no isolamento dos seguintes fungos, em ordem decrescente de frequiência: Penicillium spp. (100\%), Fusarium spp. (84\%), A. terreus (76\%), Mucor spp. (28\%), fungos nãoesporulados (F.N.E.) (20\%), Cladosporium spp. (16\%), A. flavus (8\%), A. niger (8\%) e Trichoderma spp. (8\%). Os fungos de maior incidência (A. terreus, Fusarium spp. e Penicillium spp.) apresentaram distribuição uniforme nos diferentes estágios de maturação do amendoim. Já a espécie A. flavus foi isolada do solo somente na $4^{\mathrm{a}}$ coleta $\left(5,0 \times 10^{3}\right.$ $\mathrm{UFC} / \mathrm{g}$ ), quando o fruto já estava maduro, na frequiência de $8,8 \%$ (Tabela 1). Estudos realizados em solos

Tabela 1 - Atividade de água $\left(\mathrm{A}_{\mathrm{a}}\right)$ das cascas de amendoim e do solo, frequência relativa (\%) de A. flavus e concentração de aflatoxinas em amostras de cascas de amendoim, em diferentes estágios de maturação e após a secagem.

\begin{tabular}{lcccllll}
\hline $\begin{array}{c}\text { Estágios de } \\
\text { maturação do } \\
\text { amendoim }\end{array}$ & $\begin{array}{c}\mathrm{A}_{\mathrm{a}} \\
\text { cascas }\end{array}$ & $\begin{array}{c}\mathrm{A}_{\mathrm{a}} \\
\text { Solo }\end{array}$ & $\begin{array}{c}\text { A. flavus } \\
(\%)\end{array}$ & & Aflatoxinas $\mu \mathrm{g} / \mathrm{kg}$ (amostras positivas) \\
\hline & & & & $\mathrm{AFB}_{1}$ & $\mathrm{AFB}_{2}$ & $\mathrm{AFG}_{1}$ & $\mathrm{AFG}_{2}$ \\
\hline Formação da vagem & 0,99 & 0,93 & 1,2 & $\mathrm{ND}$ & $\mathrm{ND}$ & $\mathrm{ND}$ & $\mathrm{ND}$ \\
Início da granação & 0,97 & 0,93 & -- & $\mathrm{ND}$ & $\mathrm{ND}$ & $\mathrm{ND}$ & $\mathrm{ND}$ \\
Granação & 0,96 & 0,99 & 49,0 & $218,5(1)$ & $\mathrm{D}(1)$ & $\mathrm{D}(1)$ & $\mathrm{ND}$ \\
Fruto maduro & 0,90 & 0,98 & 8,8 & $126,1(4)$ & $1,83(2)$ & $9,7(2)$ & $0,68(3)$ \\
Fruto seco & 0,61 & -- & -- & $5,42(1)$ & $0,35(1)$ & $\mathrm{ND}$ & $\mathrm{ND}$ \\
\hline
\end{tabular}

Aa: atividade de água

ND: não detectado

D: detectado 


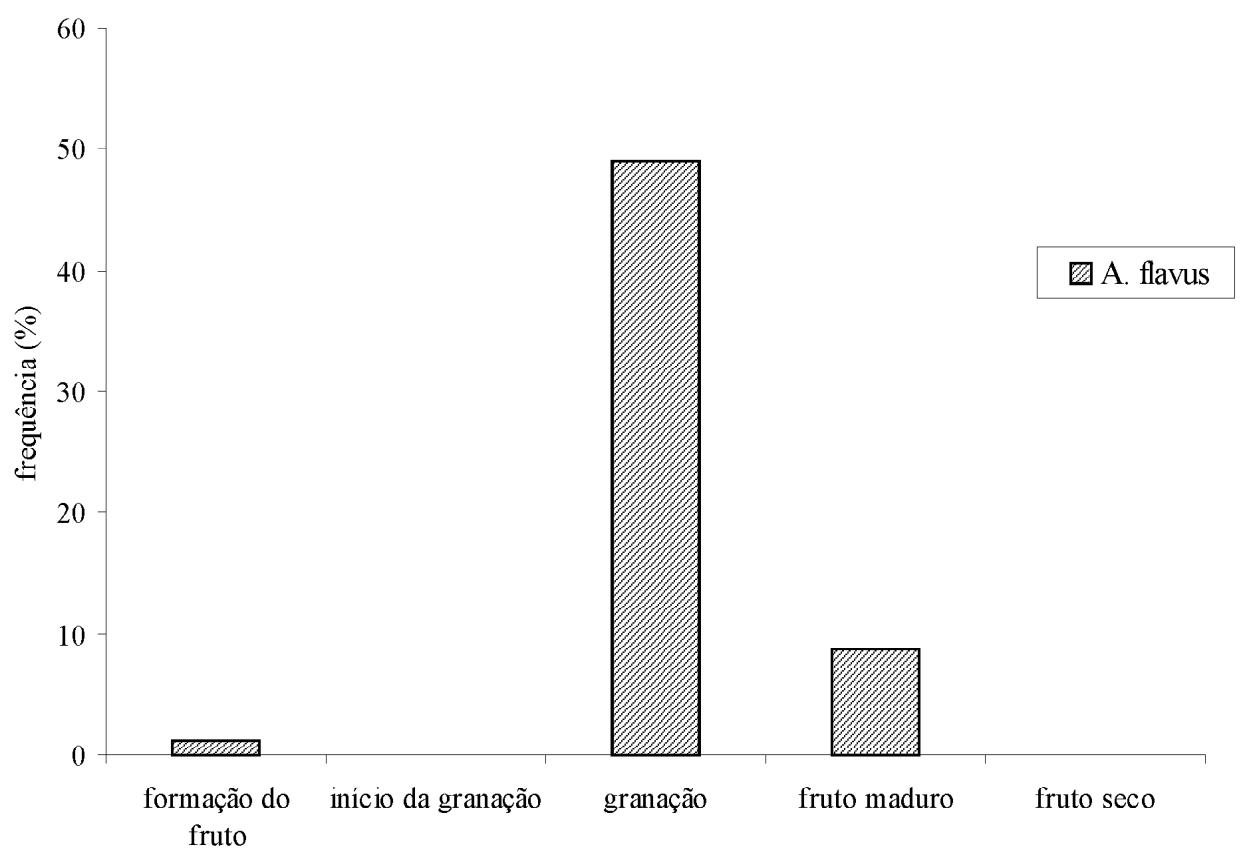

Figura 1 - Distribuição de Aspergillus flavus nas cascas de amendoim, nos diferentes estágios de maturação do fruto.

provenientes de outras regiões do Estado de São Paulo, também demonstraram Penicillium spp. como o fungo de maior prevalência (POZZI et al., 2005). Neste sentido, Klich (1998) isolou várias espécies de Penicillium, Fusarium e Aspergillus em amostras de solo no Arizona e na Califórnia. A presença de A. flavus em amostras de solo, durante a coleta dos frutos de amendoim, sinalizam esse como o reservatório primário do fungo, pois sendo o amendoim um fruto subterrâneo, suas vagens estão diretamente expostas à contaminação (HORN et al., 2005). No solo, os níveis de atividade de água registrados durante os diferentes estágios de maturação dos grãos se mantiveram acima de 0,9 (Tabela 1 ).

Os frutos-recém formados e no início da granação, constituídos por uma grande quantidade de água, não apresentaram contaminação por aflatoxinas. Dorner et al. (1989) demonstraram que a diminuição do conteúdo de água dos frutos do amendoim também reduz a capacidade desses de produzir fitoalexinas, resultando em crescimento fúngico e produção de aflatoxinas.

Os métodos para análises de aflatoxina $\mathrm{B}_{1}, \mathrm{~B}_{2}, \mathrm{G}_{1} \mathrm{e}$ $\mathrm{G}_{2}$, demonstraram os seguintes porcentuais de recuperação: $83 \%, 82 \%, 90 \%$ e $61 \%$, para as concentrações de $4,85 \mathrm{ng} /$ g, $0,60 \mathrm{ng} / \mathrm{g}, 1,52 \mathrm{ng} / \mathrm{g}$ e $1,15 \mathrm{ng} / \mathrm{g}$, respectivamente.

Das 25 amostras analisadas, 6 (24\%) apresentaram contaminação por aflatoxinas. As aflatoxinas foram detectadas em $4 \%$ das amostras do estágio de granação,
$16 \%$ dos grãos após a maturação e em 4 \% dos grãos após a secagem, em concentrações que variaram de $5,42 \mu \mathrm{g} / \mathrm{kg}$ a $218,52 \mu \mathrm{g} / \mathrm{kg}$. A aflatoxina $B_{1}$ foi detectada em $100 \%$ das amostras contaminadas e as aflatoxinas $B_{2}, G_{1}$ e $G_{2}$ foram detectadas em $83,3 \%, 50 \%$ e $50 \%$, respectivamente. Em cascas de frutos maduros, a atividade de água também foi um fator importante para contaminação por aflatoxinas $\left(\mathrm{p}<0,05\right.$ e $\left.\mathrm{r}^{2}=0,75\right)$.

A Tabela 1 indica a concentração de aflatoxinas $B_{1}$, $B_{2}, G_{1}$ e $G_{2}$ nos diferentes estágios de maturação e após a secagem das cascas de amendoim, onde se pode observar a presença de aflatoxinas em maior concentração no estágio de granação, seguido pelo fruto maduro e após a secagem. As cascas analisadas são provenientes de grãos que apresentaram contaminação por aflatoxinas (GONÇALEZ et al., 2005). A análise estatística dos resultados obtidos revelou que a presença de $A$. flavus, no solo do local do plantio, contribuiu para contaminação das cascas por aflatoxinas nos frutos maduros ( $\mathrm{p}<0,05 \mathrm{e}^{2}=0,80$ ) e que, em todos os estágios de maturação, a contaminação dos grãos e das cascas por aflatoxinas estão relacionadas ( $<<0,05$ e $\left.\mathrm{r}^{2}=0,90\right)$. Sanders et al. (1984) relataram que a contaminação de cascas de amendoim por aflatoxinas estaria relacionada com a presença da toxina nos grãos e que essa contaminação seria proveniente de partes desses presentes nas cascas. 
Os métodos para análises de ácido ciclopiazônico e fumonisinas $\mathrm{B}_{1}$ e $\mathrm{B}_{2}$ apresentaram $62 \%, 84 \%$ e $71,35 \%$ de recuperação para as concentrações $180 \mathrm{ng} / \mathrm{g}, 0,85 \mathrm{ng} / \mathrm{g}$ e $0,39 \mathrm{ng} / \mathrm{g}$, respectivamente. As análises das 25 amostras de cascas de amendoim não revelaram a presença de ácido ciclopiazônico nem das fumonisinas $\mathrm{B}_{1}$ e $\mathrm{B}_{2}$, em níveis detectáveis.

\section{CONCLUSÕES}

A contaminação das cascas de amendoim por aflatoxinas antes da colheita, representa um grande problema econômico para a indústria de alimentos e para saúde humana e animal. A introdução de processos de boas práticas agrícolas pode ser uma maneira de minimizar o problema da contaminação da vagem do amendoim por fungos toxigênicos e aflatoxinas, assim como um rígido controle de qualidade nas cascas armazenadas, uma vez que as granjas da região se utilizam desse material para confecção de cama de frango.

\section{AGRADECIMENTOS}

À FAPESP pelo financiamento do projeto e a FUNDEPAG pela bolsa concedida ao estudante Tiago N. de Souza.

\section{REFERÊNCIAS BIBLIOGRÁFICAS}

ASSOCIATION OF OFFICIAL AGRICULTURAL CHEMISTS INTERNATIONAL. AOAC official method 991.31: aflatoxins in corn, raw peanuts and peanut butter: immunoaffinity column (aflatest) method. Washington, DC, 2000. p. 49.2.18.

BARNETT, H. L.; HUNTER, B. B. Illustrated genera of imperfect fungi. Minneapolis: Burguess, 1972.

CHAN, D.; MACDONALD, S. J.; BOUGHTFLOWER, V.; BRERETON, P. Simultaneous determination of aflatoxins and ochratoxin A in food using a fully automated immunoaffinity column clean-up and liquid chromatography-fluorescence detection. Journal of Chromatography A, v. 1059, p. 13-16, 2004.

COLLINS, J. L.; POST, A. R. Peanut hull flour as a potencial source of dietary fiber. Journal of Food Science, Chicago, v. 46, p. 445-448, 451, 1981.

DELP, R. B.; STWELL, L. J.; MAROIS, J. J. Evaluation of field sampling techniques for estimation of disease incidence. Phytopathology, Saint Paul, v. 76, p. 1299-1305, 1986.
DORNER, J. W.; COLE, R. J.; SANDERS, T. H.; BLANKENSHIP, P. D. Interrelationship of kernel water activity, soil temperature, maturity, and phytoalexin production in preharvest aflatoxin contamination of drought-stressed peanuts. Mycopathologia, v. 105, p. 117$128,1989$.

FAGUNDES, M. H. Sementes de amendoim: alguns comentários. 2002. Disponível em: 〈www.conab.gov.br Acesso em: 28 ago. 2006.

GONÇALEZ, E.; NOGUEIRA, J. H. C.; FONSECA, H.; FELICIO, J. D.; ROSSI, M. H.; AQUINO, S.; SOUZA, T. N.; MANGINELLI, S.; CORRÊA, B. Análise de aflatoxinas em amendoim em diferentes estágios de maturação. In: CONGRESSO BRASILEIRO DE MICROBIOLOGIA, 23., 2005, Santos, SP. Anais... Santos, 2005. p. 16/412-1.

HORN, B. W. Colonization of wounded peanuts seeds by soil fungi: selectivity for species from Aspergillus section Flavi. Mycologia, v. 97, p. 202-217, 2005.

KLICH, M. A. Soil fungi of some low-altitude desert cotton fields and ability of their extracts to inhibit Aspergillus flavus. Mycopathologia, v. 142, p. 97-100, 1998.

LIEN, R. J.; HESS, J. B.; CONNER, D. E.; WOOD, C. W.; SHELBY, R. A. Peanut hulls as a litter source for broiler breeder replacement pullets. Poultry Science, Champaign, v. 77, p. 41-46, 1998.

LISKER, N.; MICHAELI, R.; FRANK, Z. R. Mycotoxigenic potential of Aspergillus flavus strains isolated from groundnuts growing in Israel. Mycopathologia, v. 122, p. 177-183, 1993.

LOSITO, I.; MONACI, L.; ARESTA, A.; ZAMBONIN, C. G. LC-ion trap eletrospray MS-MS for detrmination of cyclopiazonic acid in milk samples. Analyst, p. 499-502, 2002.

MARTINS, R. Amendoim: monitoramento da cadeia de produção. Análise e Indicadores dos Agronegócios, São Paulo, v. 1, n. 1, 2006. Disponível em: <www.iea.sp.gov.bry. Acesso em: 28 ago. 2006a.

MARTINS, R. Amendoim: perspectivas de aumento da produtividade até $\mathbf{2 0 1 0}$. Análise e Indicadores dos Agronegócios, São_Paulo, v. 1, n. 6, 2006. Disponível em: uWwW.iea.sp.gov.bri '>. Acesso em: 28 ago. 2006 b. 
MARTINS, R. Amendoim: perspectivas para a safra 2007/ 08. Análise e Indicadores dos Agronegócios, São Paulo, v. 2, n. 9, 2007. Disponível em: 〈wWw.iea.sp.gov.br〉. Acesso em: 28 jan. 2008.

MPHANE, F. A.; SIAME, B. A.; TAYLOR, J. E. Fungi, aflatoxin and cyclopiazonic acid associated with peanut retailing in Botswana. Journal of Food Protection, Des Moines, v. 67, p. 96-102, 2004.

NELSON, P. E.; TOUSON, T. A.; MARASAS, W. F. O. Fusarium species. An illustrated manual for identification. London: The Pennsylvania State University, 1983.

PITT, J. I.; KING, A. D.; HOCKING, A. D. Dchloran-rose Bengal medium for enumeration and isolation of molds from foods. Applied and Environmental Microbiology, Washington, v. 37, p. 959-970, 1979.

POZZI, C. R.; BRAGHINI, R.; ARCARO, J. R.; ZORZETE, P.; POZAR, I. O.; DENUCCI, S.; CORREA, B. Mycoflora and occurrence of alternariol and alternariol monomethyl ether in Brazilian sunflower from sowing to harvest. Journal of Agricultural and Food Chemistry, Easton, v. 53 , n. 14 , p. $5824-5828,2005$.

RAPER, K. B.; FENNEL, D. I. The genus aspergillus Baltimore: Willians \& Wilkins, 1965.

SANDERS, T. H.; MCMEANS, J. L.; DAVIDSON, J. I. Aflatoxin content of peanut hulls. Journal of the American Oil Chemists'Society, Chicago, v. 61, n. 12, p. 1839-1841, 1984.

SANGARE-TIGORI, B.; MOUKHA, S.; KOUADIO, H. J.; BETBEDER, A. M.; DANO, D. S.; CREPPY, E. E. Co- occurrence of aflatoxin b1, fumonisin b1, ochratoxin A and zearalenone in cereals and peanuts from Côte d'Ivoire. Food Additives and Contaminants, v. 23, n. 10, p. 1000-1007, 2006.

SAS INSTITUTE. SAS/STAT user's guide: the REG procedure. Disponível em! <http://Www.asu.edu/sash isasdoc/sashtml/stat/chap55/index.htm>.' Acesso em: 14 mar. 2008

SMITH, J. E.; MOSS, M. O. Mycotoxins, formation, analysis and significance. New York: J. Wiley \& Sons, 1985.

SWANSON, K. M.; BUSTA, F. F.; PETTERSON, E. H.; JOHNSON, M. G. Colony count methods. In: VANDEZANT, C.; SPLITTOESSER, D. S. (Eds.). Compendium of methods for the microbiological examination of foods. New York: American Public Health Association, 1992.

TARIN, A.; ROSELL, M. G.; GUARDINO, X. Use of highperformance liquid chromatography to assess airbone mycotoxins aflatoxins and ochratoxin A. Journal of Chromatography A, v. 1047, p. 235-240, 2004.

URANO, T.; TRUCKSESS, M. W.; BEAVER, R. W.; WILSON, D. M.; DORENER, J. W.; DOWELL, F. E. Cooccurrence of cyclopiazonic acid and aflatoxins in corn and peanuts. Journal of Association of Official Agricultural Chemists International, v. 75, p. 838-841, 1992.

VISCONTI, A.; SOLFRIZZO, M.; GIROLAMO, A. Determination of fumonisins $\mathrm{B}_{1}$ and $\mathrm{B}_{2}$ in corn and corn flakes by liquid chromatography with immunoaffinity column cleanup: collaborative study. Food Chemical Contaminants, v. 84, p. 1828-1837, 2001. 